\title{
Cornering the Fear Engram: Long-Term Synaptic Changes in the Lateral Nucleus of the Amygdala after Fear Conditioning
}

\author{
Jeong-Tae Kwon and June-Seek Choi \\ Department of Psychology, Korea University, Seoul 136-701, Korea
}

Use-dependent synaptic modifications in the lateral nucleus of the amygdala (LA) have been suggested to be the cellular analog of memory trace after pavlovian fear conditioning. However, whether neurophysiological changes in the LA are produced as a direct consequence of associative learning awaits additional proof. Using microstimulation of the medial geniculate nucleus of the thalamus as the conditioned stimulus (CS), we demonstrated that contingent pairings of the brain-stimulation CS and a footshock unconditioned stimulus lead to enhanced synaptic efficacy in the thalamic input to the LA, supporting the hypothesis that localized synaptic alterations underlie fear memory formation.

\section{Introduction}

Despite the near-unanimous consensus that learning induces detectable synaptic modifications in the brain, as originally proposed by Hebb (1949) and formularized in the "synaptic plasticity and memory (SPM)" hypothesis (Martin et al., 2000), direct evidence linking localized cellular changes with behavior has been rare. In mammalian brain, complexity of most learning tasks unavoidably demands a network of neurons, making it impossible to monitor all potentially relevant synapses. A prerequisite for testing the SPM hypothesis, therefore, is to identify the neural circuit for the target behavior and to focus on the key sites for learning-induced synaptic plasticity. Pavlovian fear conditioning is among a handful of learning paradigms in which well defined neural circuits have been delineated.

In a typical fear conditioning, an initially neutral conditioned stimulus (CS) is contingently paired with an aversive unconditioned stimulus (US). As a result, the CS comes to elicit the conditioned response (CR), an orchestration of autonomic, behavioral, and endocrine responses that prepares the organism for the upcoming threat. The fast and reliable nature of fear conditioning allowed an extensive analysis of related neural components in mammalian brain (Blanchard and Blanchard, 1972; Davis, 1992; LeDoux, 2000). The weight of the anatomical and neurobiological evidence suggests that the amygdala, especially the lateral nucleus (LA), is where the sensory information for the CS and the US converge (Romanski et al., 1993), and the critical cellular changes occur as a result of the convergent activation (Fanselow and LeDoux, 1999).

A plausible hypothesis for the cellular mechanisms of fear memory trace has been proposed to suggest that neurophysiological

Received Dec. 6, 2008; revised May 12, 2009; accepted June 24, 2009.

This research was supported by a grant (M103KV010025-07K2201-02510) from the Brain Research Center of the 21st Century Frontier Research Program funded by the Ministry of Science and Technology, the Republic of Korea. We thank Eun Joo Kim and Junchol Park for help with running the experiments.

Correspondence should be addressed to June-Seek Choi, Department of Psychology, Korea University, 5-1 Anam, Seongbuk, Seoul 136-701, Korea. E-mail: j-schoi@korea.ac.kr.

DOI:10.1523/JNEUROSCI.5928-08.2009

Copyright $\odot 2009$ Society for Neuroscience $\quad$ 0270-6474/09/299700-04\$15.00/0 properties of LA neurons change as a result of learning (Maren, 1999; Blair et al., 2001). Specifically, fear conditioning potentiates synaptic efficacy of some LA neurons, and the increased excitability of those neurons modifies the way CS information is transmitted. In support of the hypothesis, it has been shown that fear conditioning enhances auditory CS-evoked field potentials (EFPs) in the LA (Rogan et al., 1997) and short-latency neuronal responses in freely moving animals (Quirk et al., 1995). Likewise, synaptic currents in LA neurons were potentiated in brain slices prepared from fear-conditioned rats (McKernan and Shinnick-Gallagher, 1997). None of the studies, however, can exclude the possibility that the synaptic changes observed in the LA are secondary to those in other synapses in the fear circuit. In fact, an alternative site of critical plasticity has been proposed (Cahill et al., 1999).

In the current study, we tested whether fear conditioning induces detectable changes in the LA using microstimulation of the medial division of the medial geniculate nucleus of the thalamus (MGm), an immediate afferent input to the LA, as the CS. Intracranial stimulation has been used as a CS to train conscious animals in eyeblink conditioning and to track converging sensory pathways (Steinmetz et al., 1986). Using direct stimulation of the MGm in fear conditioning provides several distinct advantages over conventional auditory CSs for detecting learning-induced cellular changes. First, MGm-stimulation CS reaches the LA directly, and any influence from upstream structures can be excluded. Second, by using the same stimulation site for both fear conditioning and triggering EFPs in the LA, the link between acquisition of memory and changes in synaptic efficacy can be more directly evaluated. In addition, our experimental design with chronically implanted stimulating electrodes allows repeated measurements of EFPs, an ideal approach for monitoring cellular changes before and after learning.

\section{Materials and Methods}

Subjects. Male Sprague Dawley rats (260-320 g; Orient Bio) were housed individually. They were maintained on a $12 \mathrm{~h}$ light/dark cycle with access to food and water ad libitum. All behavioral procedures were conducted during the light phase of the cycle. 
a

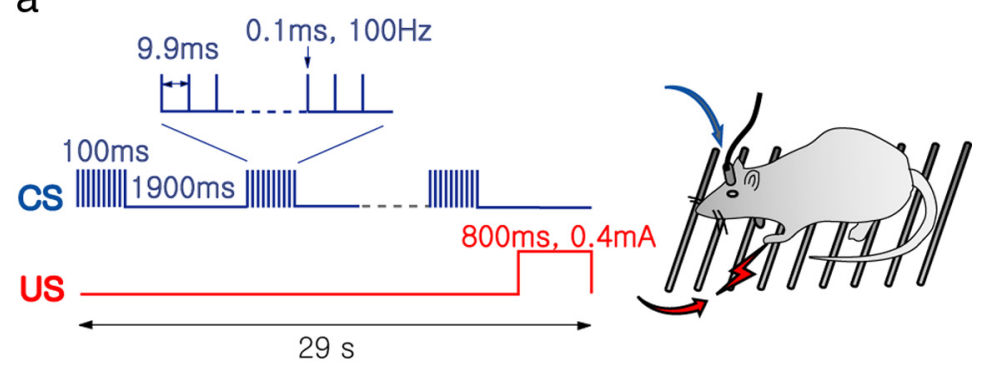

b

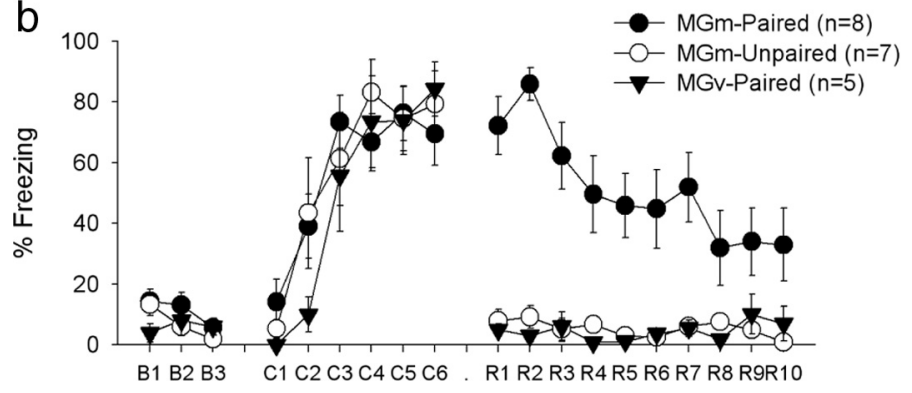

Figure 1. Fear conditioning with brain-stimulation CSs. $\boldsymbol{a}$, Stimuli configuration. The $\mathrm{CS}$ was intermittent trains of brain stimulation (15 trains of $100.1 \mathrm{~ms}$ square pulses), which was paired with the footshock US during the conditioning phase. $\boldsymbol{b}$, Changes in freezing during conditioning. During the preconditioning phase (B1-B3), none of the three groups showed any freezing to the $\mathrm{CS}$. During the conditioning phase (C1-C6), significant increase in freezing was observed in all three groups. During the retention test (R1-R10), however, there was a significant group difference. Only the MGm-paired group showed significant freezing on retention compared with the preconditioning baseline. Freezing was essentially absent in the other two groups.

EFP measurement and analysis. Two EFP sessions were administered $24 \mathrm{~h}$ before and after the conditioning under anesthesia with sodium pentobarbital (60 mg/kg, i.p.). The head was fixed onto the stereotaxic frame, and a monopulse stimulus $(100 \mu \mathrm{s})$ was delivered through the stimulating electrode while EFP was being monitored in the LA. The stimulus intensity was set at $50 \%$ of the current that produces the maximum EFP (0.1-1 mA). Once the intensity was determined, stimulation was delivered at every $30 \mathrm{~s}$ for $15 \mathrm{~min}$.

EFP was amplified $(1000 \times)$ by an alternating current amplifier (model 1800; A-M Systems), bandpass filtered ( $1 \mathrm{~Hz}$ to $1 \mathrm{kHz}$ ), and digitized at $10 \mathrm{kHz}$ to be stored in the computer. Recorded signals were analyzed by a custom-made program (Labview; National Instruments) to measure the peak-to-valley amplitude (potential difference between the first positive peak and negative valley). The peak and the valley were defined as the maximum and minimum potential value within given time windows (1-3 ms for the peak and 3-10 ms for the valley). The latency was defined as the time to the first valley. Amplitudes of EFP were normalized to the preconditioning baseline values.

\section{Results}

\section{Experiment 1: fear conditioning with} the brain-stimulation CS

Surgery for electrode implantation. Rats were deeply anesthetized with sodium pentobarbital (60 mg/kg, i.p., supplemented as necessary) and placed on a stereotaxic instrument (David Kopf Instruments). Bipolar stimulating electrodes (125 $\mu \mathrm{m}$; MS303-3-B; Plastics One) were implanted in the MGm [ $-5.8 \mathrm{~mm}$ anteroposterior (AP); $2.9 \mathrm{~mm}$ mediolateral (ML) from bregma; 5.5-6.0 mm below dura] or the ventral division of the medial geniculate nucleus (MGv) $(-5.8 \mathrm{~mm} \mathrm{AP} ; 3.5 \mathrm{~mm}$ ML from bregma; 5.5-6.0 mm below dura) based on Paxinos and Watson (1998). For experiment 2, recording electrodes (tungsten, $5 \mathrm{M} \Omega$; A-M Systems) were also implanted in the ipsilateral LA. For the placement of the recording electrode, a standard coordinate $(-3.3 \mathrm{~mm}$ AP; $5.5 \mathrm{~mm} \mathrm{ML}$; $5.5-6.0 \mathrm{~mm}$ below dura) was used, with additional adjustment provided by MGm stimulation. The final depth of the recording electrode was determined so that EFP was at maximum.

Conditioning procedure. The CS was produced by a pulse generator (MASTER-8; A.M.P.I.) and delivered through a stimulus isolator (ISO-Flex; A.M.P.I.) to the stimulating electrode connected by a captive collar (Plastics One).

On the first day of training, the stimulation intensity for the CS was determined by detecting observable orienting responses, such as ear pricking or head movements. Stimulus intensity was set at $30 \mu \mathrm{A}$ initially and gradually increased until the rat showed one of the orienting responses. Once the final current was determined, $\sim 80 \%$ of that level was used for the CS throughout the experiment. The current levels ranged between 30 and $200 \mu \mathrm{A}$. On the second day, the baseline freezing was measured to three CS-only trials. On the third day, the subjects received six paired or unpaired conditioning trials with the brain stimulation CS and the footshock US (for the stimulation parameters, refer to Fig. 1). Then on the fourth day, they were placed in a new context and presented with 10 retention trials. The context differ in several features: flat floor with wood shavings instead of the grid, cylindrical wall instead of square, and blue instead of red illumination. In experiment 2, conditioning procedure was essentially identical to experiment 1 , except that a recording session was inserted on days 3 and 5 , extending the whole experiment to 6 rather than $4 \mathrm{~d}$. Movement was recorded through an infrared video camera mounted in the ceiling and analyzed offline. Freezing was defined as no movements except for breathing. Freezing data was analyzed using repeated measures ANOVA with session as a within-subject variable and conditioning group as a between-subject variable.
In the first experiment, we tested whether electrical stimulation of the MGm or MGv could be used as the CS. Rats implanted with a bipolar stimulating electrode were subjected to fear conditioning in which a train of brain stimulation was paired with the footshock US (Fig. 1a). Rats in the MGm-unpaired group received the same number of CSs and USs but in a noncontingent manner. Freezing was measured as the CR. Repeated measures ANOVA revealed significant main effects of group $\left(F_{(2,17)}=\right.$ 13.678; $p<0.01)$ and session $\left(F_{(2,34)}=51.639 ; p<0.01\right)$, as well as interaction $\left(F_{(4,34)}=11.650 ; p<0.01\right)$. To further examine the group difference, we conducted one-way ANOVA in each session. During the preconditioning session, none of the stimulation groups showed any significant freezing to the CS $\left(F_{(1,19)}=1.008\right.$; $p=0.386)$. During the conditioning session, all three groups showed a high level of freezing, a typical immediate response to aversive footshock. During the retention test, however, there was a significant group difference $\left(F_{(2,17)}=33.909 ; p<0.01\right)$. Post hoc test with Bonferroni's correction revealed that the MGm-paired group froze significantly more to the CS than the MGm-unpaired or MGv-paired group did ( $p$ values $<0.001$ ) (Fig. 1b). These data indicate that the MGm stimulation can function as an effective CS to induce normal fear CR. The level of freezing was comparable with that obtained from auditory fear conditioning experiments with similar parameters in our laboratory. The CR was produced by the associative learning rather than nonspecific sensitization because neither brain stimulation alone nor noncontingent pairing of the CS and the US resulted in a significant level of freezing.

The locus of stimulation was critical, because the MGv-paired group showed no CR despite the fact that they underwent exactly the same conditioning procedure, and the MGv stimulation site was only $0.5 \mathrm{~mm}$ away from the MGm. The lack of CR with MGv stimulation indicates that MGm stimulation is privileged in producing learning-related changes after fear conditioning, perhaps attributable to direct thalamo-amygdala projections (Fig. 1b). 
Unlike MGm, MGv does not have direct projection to the LA. Instead, it mainly provides input to the auditory cortex (Romanski and LeDoux, 1993). Interestingly, similar functional dissociation of MGm and MGv was found in eyeblink conditioning (Steinmetz et al., 1986; Campolattaro et al., 2007).

\section{Experiment 2: enhanced EFP in the LA after fear conditioning}

In the second experiment, we tested whether conditioning with the MGmstimulation CS could induce synaptic changes. As an index of synaptic efficacy, EFP in the MGm-LA synapse was measured. In addition to the stimulating electrode in the MGm, a recording electrode was also implanted in the LA. Conditioning and testing procedures were identical to experiment 1 , except that recording sessions were inserted before and after the fear conditioning (Fig. 2a). EFP was measured under anesthesia. Before the conditioning, there was no significant difference between the MGm-paired and MGm-unpaired groups in the baseline amplitude of the EFP to the monopulse stimulation of the MGm (MGm-paired group, $255.3 \pm 32.6$ $\mathrm{mV}$; MGm-unpaired group, 227.3 \pm 36.9 $\mathrm{mV} ; p=0.459$, independent $t$ test). Twenty-four hours after the conditioning, however, only the MGm-paired group showed significantly higher level of EFP to the same MGm stimulation compared with the preconditioning session $(p<$ 0.01 , paired $t$ test), whereas the MGmunpaired group did not $(p=0.592$, paired $t$ test) (Fig. $2 d, f$ ). Consistent with experiment 1 , conditioning also induced a significant level of freezing to the CS in the MGm-paired group when tested $48 \mathrm{~h}$ after the conditioning session $\left(F_{(1,13)}=12.351\right.$; $p<0.01$, repeated measures ANOVA) (Fig. $2 b$ ). Significant correlation was observed between freezing during the retention test and EFP enhancement $(R=0.722 ; p<0.05)$ (Fig. $2 g)$, providing strong evidence that the increased responding to MGm stimulation is the result of conditioning and acquired fear memory. There was no significant change in the latency to the first negative valley after the enhancement, indicating that the EFP is perhaps monosynaptic (preconditioning, $6.27 \pm 0.34 \mathrm{~ms}$; postconditioning, $6.13 \pm 0.27 \mathrm{~ms}$, mean $\pm \mathrm{SEM} ; p=0.462$, paired $t$ test $)$.

\section{Discussion}

The current study contributes a unique set of data toward the search for the fear engram. Using local brain stimulation as the $\mathrm{CS}$, we demonstrated that conditioning-related synaptic plasticity in the LA can be induced at the efferent of the thalamus. Activation of the thalamic input to the LA, contingently paired with an aversive US in a conscious behaving animal, was sufficient to induce enhanced synaptic strength that was highly correlated with subsequently measured fear, indicating that fear memory trace is
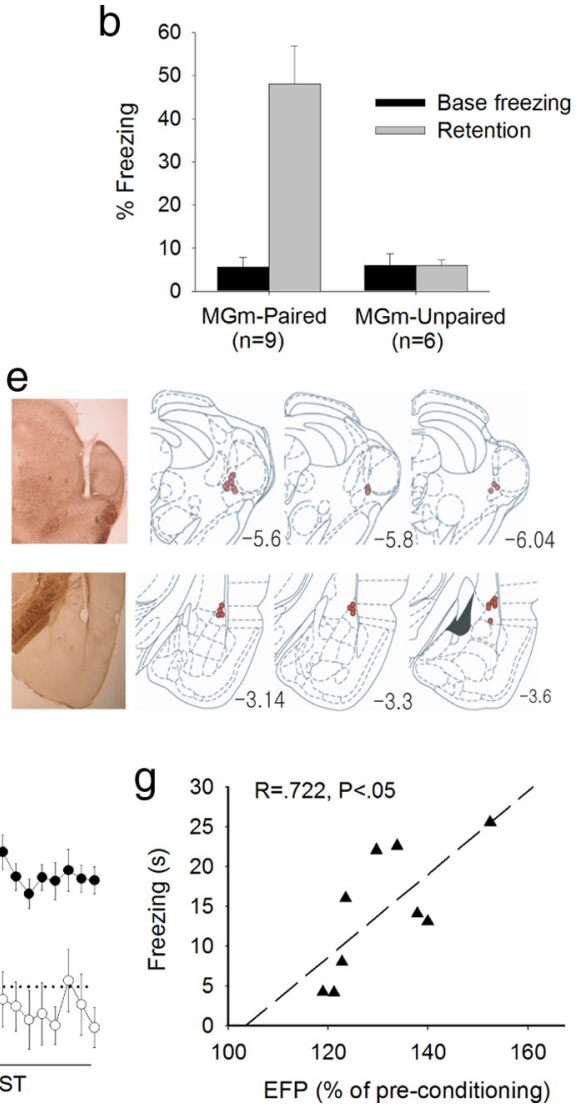

Figure 2. Enhanced EFP in the LA after fear conditioning. $\boldsymbol{a}$, Experimental procedure for experiment 2. The procedure is identical waveform on a single trial (gray line, preconditioning; black line, postconditioning). EFP amplitude was defined as the difference between the first positive peak and the first negative valley, marked by two dotted horizontal lines. Calibration: $200 \mu \mathrm{V}, 5 \mathrm{~ms}$ $\boldsymbol{e}$, Electrode placement. Photos show stimulating (top) and recording (bottom) sites, marked by small electrolytic lesions at the tip. subdivision of the LA. The drawings were modified from Paxinos and Watson (1998). $\boldsymbol{f}$, Average EFP data showing percentage 列 ifference. In addition, the MGm-paired group also showed greater EFP than the MGm-unpaired group during the postconditioning test. $\boldsymbol{g}$, Relation between fear memory retention and postconditioning EFP enhancement.

stored as a form of modified synaptic efficacy (Martin et al., 2000; Blair et al., 2001).

Previous studies that have shown enhanced response in the LA after fear conditioning had limited implications attributable to the use of external sensory stimuli (Quirk et al., 1995; Rogan et al., 1997; Goosens et al., 2003). Although these studies reached the same conclusion by measuring various aspects of cellular property, they cannot exclude the possibility that the enhanced neural response to the CS was caused by changes in the sensory pathways upstream of the LA. For example, synaptic changes within the MGm itself have been credited for the formation of fear memory and enhanced synaptic efficacy in the LA (Edeline and Weinberger, 1992). Another study has also attempted to isolate a localized change by recording synaptic currents in brain slices obtained from fear-conditioned rats $24 \mathrm{~h}$ after the memory retention test. EPSCs to the direct stimulation of the thalamic input were potentiated in the paired conditioning but not in the unpaired or naive control groups (McKernan and ShinnickGallagher, 1997). Although in vitro preparation measures a highly localized synaptic response, it still does not guarantee that the en- 
hanced synaptic efficacy in the LA is independent of any upstream plasticity, which might be formed and promote LA plasticity before the slice is prepared. Schafe et al. (2005) have complemented the previous studies using site-specific pharmacological manipulation. Intra-LA infusions of a drug that blocks intracellular signaling cascades necessary for synaptic modification, impaired long-term retention of fear memory, and potentiation of auditory EFPs in the LA but not in the MGm. Consistent with the preceding studies, our results strongly argue that the enhanced synaptic transmission in the thalamic input to the LA is a direct consequence of fear conditioning. Because we adopted a within-subject design in which MGm-LA synaptic efficacy was repeatedly measured before and after fear conditioning, individual variations had little effect. The differences in EFP enhancements between conditioning and control groups must result from associative pairings of the CS and US. A strong correlation was found between the magnitude of synaptic strength and the amount of freezing, further supporting the argument that learning induces synaptic potentiation in the LA. In our design, synaptic strength was measured before the behavioral $\mathrm{CR}$ was measured in the retention test, suggesting that the synaptic potentiation might be causally related to the freezing level.

The reduced fear circuit involved in conveying the fear memory trace in the current design rendered the neurophysiological change more easily identified and left little possibility for an alternative interpretation. However, there are still multiple candidate sites of plasticity, especially along the efferent projections from the LA. For example, LA activation is transmitted to the brainstem circuit via the central nucleus (CE), and the CE itself also has been implicated in hosting neural plasticity of fear memory. Because all connections among all amygdala subnuclei have been left intact, we cannot exclude the possibility that fear conditioning induced synaptic modifications at the output stream of the LA (Koo et al., 2004; Samson and Paré, 2005).

Stimulation of posterior intralaminar thalamic nucleus (PIN), a neighboring region of $\mathrm{MGm}$, has been known to relay somatosensory information, especially a pain-related signal. It is possible that MGm stimulation alone is sufficient for the induction of the conditioned response because of adjacent PIN activation. A previous study reported that PIN stimulation alone evoked freezing and functioned as the US for fear conditioning (Cruikshank et al., 1992). We also observed freezing in MGm-stimulated rats when the intensity was high (data not shown). However, at the intensity level used in the current study, none of the stimulated rats showed any freezing during the preconditioning session or on the first trial of the conditioning session (Fig. 1b). Conversely, stimulation of the MGv, which is only $0.5 \mathrm{~mm}$ away from the MGm, was not effective in inducing fear CR or reliable EFP (supplemental Fig. 1, available at www.jneurosci.org as supplemental material). Most importantly, the MGm-unpaired group did not show any significant amount of freezing even after repeated stimulation unpaired with the US, indicating that the fear CR is not the result of nonspecific sensitization induced by the aversive footshock.

In short, combining a simple associative learning with the well established brain circuit, we confirmed the SPM hypothesis in fear conditioning that the use-dependent synaptic plasticity in LA is induced as a direct consequence of contingent pairings of the CS and US in behaving animals. High correlation was found between the increased EFPs in LA neurons and the CR level, strongly implying a causal relationship. Additional study is needed to test whether MGm-LA synaptic plasticity is sufficient for fear memory storage.

\section{References}

Blair HT, Schafe GE, Bauer EP, Rodrigues SM, LeDoux JE (2001) Synaptic plasticity in the lateral amygdala: a cellular hypothesis of fear conditioning. Learn Mem 8:229-242.

Blanchard DC, Blanchard RJ (1972) Innate and conditioned reactions to threat in rats with amygdaloid lesions. J Comp Physiol Psychol 81:281-290.

Cahill L, Weinberger NM, Roozendaal B, McGaugh JL (1999) Is the amygdala a locus of "conditioned fear"? Some questions and caveats. Neuron 23:227-228.

Campolattaro MM, Halverson HE, Freeman JH (2007) Medial auditory thalamic stimulation as a conditioned stimulus for eyeblink conditioning in rats. Learn Mem 14:152-159.

Cruikshank SJ, Edeline JM, Weinberger NM (1992) Stimulation at a site of auditory-somatosensory convergence in the medial geniculate nucleus is an effective unconditioned stimulus for fear conditioning. Behav Neurosci 106:471-483.

Davis M (1992) The role of the amygdala in fear and anxiety. Annu Rev Neurosci 15:353-375.

Edeline JM, Weinberger NM (1992) Associative retuning in the thalamic source of input to the amygdala and auditory cortex: receptive field plasticity in the medial division of the medial geniculate body. Behav Neurosci 106:81-105.

Fanselow MS, LeDoux JE (1999) Why we think plasticity underlying Pavlovian fear conditioning occurs in the basolateral amygdala. Neuron 23:229-232.

Goosens KA, Hobin JA, Maren S (2003) Auditory-evoked spike firing in the lateral amygdala and Pavlovian fear conditioning: mnemonic code or fear bias? Neuron 40:1013-1022.

Hebb DO (1949) The organization of behavior. New York: Wiley.

Koo JW, Han JS, Kim JJ (2004) Selective neurotoxic lesions of basolateral and central nuclei of the amygdala produce differential effects on fear conditioning. J Neurosci 24:7654-7662.

LeDoux JE (2000) Emotion circuits in the brain. Annu Rev Neurosci 23:155-184.

Maren S (1999) Long-term potentiation in the amygdala: a mechanism for emotional learning and memory. Trends Neurosci 22:561-567.

Martin SJ, Grimwood PD, Morris RG (2000) Synaptic plasticity and memory: an evaluation of the hypothesis. Annu Rev Neurosci 23:649-711.

McKernan MG, Shinnick-Gallagher P (1997) Fear conditioning induces a lasting potentiation of synaptic currents in vitro. Nature 390:607-611.

Paxinos G, Watson C (1998) The rat brain in stereotaxic coordinates. San Diego: Academic.

Quirk GJ, Repa C, LeDoux JE (1995) Fear conditioning enhances shortlatency auditory responses of lateral amygdala neurons: parallel recordings in the freely behaving rat. Neuron 15:1029-1039.

Rogan MT, Stäubli UV, LeDoux JE (1997) Fear conditioning induces associative long-term potentiation in the amygdala. Nature 390:604-607.

Romanski LM, LeDoux JE (1993) Organization of rodent auditory cortex: anterograde transport of PHA-L from MGv to temporal neocortex. Cereb Cortex 3:499-514.

Romanski LM, Clugnet MC, Bordi F, LeDoux JE (1993) Somatosensory and auditory convergence in the lateral nucleus of the amygdala. Behav Neurosci 107:444-450.

Samson RD, Paré D (2005) Activity-dependent synaptic plasticity in the central nucleus of the amygdala. J Neurosci 25:1847-1855.

Schafe GE, Doyère V, LeDoux JE (2005) Tracking the fear engram: the lateral amygdala is an essential locus of fear memory storage. J Neurosci 25:10010-10014.

Steinmetz JE, Rosen DJ, Chapman PF, Lavond DG, Thompson RF (1986) Classical conditioning of the rabbit eyelid response with a mossy-fiber stimulation CS. I. Pontine nuclei and middle cerebellar peduncle stimulation. Behav Neurosci 100:878-887. 